\title{
Lipidomic analysis of human plasma reveals ether-linked lipids that are elevated in morbidly obese humans compared to lean
}

\author{
Elise L Donovan ${ }^{1,3^{*}}$, Stefan M Pettine ${ }^{2}$, Matthew S Hickey ${ }^{1}$, Karyn L Hamilton ${ }^{1}$ and Benjamin F Miller ${ }^{1}$
}

\begin{abstract}
Background: Lipidomic analysis was performed to explore differences in lipid profiles between plasma from lean and obese subjects, followed by in vitro methods to examine a role for the identified lipids in endothelial cell pathophysiology.

Methods: Plasma was collected from 15 morbidly obese and 13 control subjects. Lipids were extracted from plasma and analyzed using LC/MS, and MS/MS to characterize lipid profiles and identify lipids that are elevated in obese subjects compared to lean.

Results: Orthogonal partial least squares-discriminant analysis (OPLS-DA) modelling showed that lipid profiles were significantly different in obese subjects compared to lean. Analysis of lipids that were driving group separation in the OPLS-DA model and that were significantly elevated in the obese group led to identification of a group of ether-linked phosphatidylcholine (PC) and phosphatidylethanolamine (PE) lipids of interest. Treatment of human coronary artery endothelial cells with the ether-linked phosphatidylethanolamine induced expression of cell adhesion molecules, a hallmark of endothelial cell activation. However, oxidized phosphatidylcholine products that can induce endothelial cell activation in vitro, were not significantly different between groups in vivo.
\end{abstract}

Conclusion: These data suggest a role for ether-linked lipids in obesity associated dyslipidemia and vascular disease.

Keywords: Lipidomics, Obesity, Dyslipidemia, Endothelial cells, Oxidized phospholipids

\section{Background}

Dyslipidemia and oxidative stress are characteristic of obesity and diabetes, and appear to play a major role in diabetes, cardiovascular disease, and cancer [1,2]. Obesity is associated with increased circulating lipids, and increased oxidized LDL that leads to inflammation and oxidative stress in vascular endothelial cells and contributes to the pathophysiology of the metabolic syndrome and atherosclerosis [3,4]. Ectopic fat deposition in morbidly obese individuals also contributes to the pathogenesis of vascular and metabolic disturbances by inhibiting insulin action and disrupting lipid metabolism in the adipose, liver, pancreas, kidney and skeletal,

\footnotetext{
* Correspondence: e.donovan@auckland.ac.nz

'Department of Health and Exercise Science, Colorado State University, Fort Collins, CO 8052, USA

${ }^{3}$ The Liggins Institute, University of Auckland, 85 Park Rd Grafton, Auckland NZ 1142, New Zealand

Full list of author information is available at the end of the article
}

smooth and cardiac muscle [5]. Phosphatidylcholine (PC) species that contain polyunsaturated fatty acids, particularly arachidonate, at the sn-2 position are especially susceptible to free radical oxidation [6]. One such PC is 1palmitoyl-2-arachidonoyl-sn-glycero-3-phosphorylcholine (PAPC), which is a common cell membrane constituent, and circulates within cholesterol particles. PAPC and several products of PAPC oxidation including 1palmitoyl-2-glutaroyl-sn-glycero-3-phosphorylcholine (PGPC), 1-palmitoyl-2-(5,6-epoxyisoprostane $E_{2}$ )-snglycero-3-phosphorylcholine (PEIPC),1-palmitoyl-2oxovaleroyl-sn-glycero-3-phosphorylcholine (POVPC), and lysophosphatidylcholine (lyso-PC) have been shown to induce cell adhesion molecule expression and inflammatory mediator secretion in endothelial cells and have been implicated in atherosclerotic progression [7-12], and oxidized phospholipids are found deposited in atherosclerotic plaques $[6,13]$. Because PC is the most

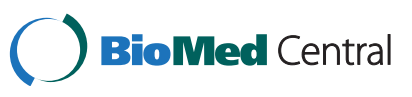


abundant cellular phospholipid, most oxidation products detected contain the choline head group [14]. However, other lipid classes and additional oxidized phospholipids are likely involved in vascular pathology, and it is likely that interactive effects between lipids contribute to $\mathrm{CVD} /$ metabolic disease pathophysiology.

In addition to (oxidized) phospholipids, other lipids including but not limited to saturated and polyunsaturated fatty acids and ether-linked lipids have been examined in the relation to metabolic syndrome and cardiovascular disease. Ether-linked lipids, characterized by an ether linkage between the glycerol backbone and one or both fatty acid side chains (usually the sn-1 position) as opposed to an ester linkage, and plasmalogens, a subclass of ether-linked lipids characterized by a vinyl ether linkage at the sn-1 position with an ester linkage at the sn-2 position, have also been the subject of recent research. Plasmalogens may function as free radical scavengers, and ether-linked lipids can serve as arachidonic acid reservoirs in cell membranes [15-17]. The role of ether-linked lipids and plasmalogens in metabolic and cardiovascular disease is still not well understood as there is lack of concensus in the data as to patterns of change in ether-linked lipid distribution with different metabolic syndrome characteristics $[18,19]$.

Lipidomics is broadly defined as the large-scale study of pathways and networks of cellular lipids in biological systems. Lipidomics can be used to examine the presence and structure of the range of lipid species within different tissues making it an attractive method for examining distribution of phospholipids in tissue, as well as for globally examining differences in lipids between subject groups. A broad examination of lipid species that are elevated and potentially contribute to metabolic and cardiovascular disease pathologies in morbidly obese humans could provide insight into the link between obesity and pathogenesis of atherosclerosis and facilitate discovery of lipid biomarkers that increase disease risk.

The purpose of this study was to: 1) use a shotgun lipidomics approach to examine global lipid distribution in plasma from lean and morbidly obese humans, to identify lipids that are elevated in the morbidly obese population that may contribute to metabolic and cardiovascular pathology, 2) examine the effects of lipids identified in our shotgun analysis as elevated in obese subjects in an in vitro endothelial cell model to determine whether they induce disease related phenotypic changes, and 3) use a targeted lipidomics approach to determine if there is a difference in distribution of oxidized phospholipids in plasma from lean and morbidly obese humans. We hypothesized that lipid profiles would be significantly different in plasma from morbidly obese humans compared to lean, and that lipids identified in our shotgun approach that are elevated in obese subjects compared to lean would induce inflammatory changes in endothelial cells. In addition, we hypothesized that oxidized phospholipids shown previously in vitro to cause endothelial cell production of inflammatory mediators and cell adhesion molecules would be greater in plasma from obese humans compared to lean.

\section{Materials and methods}

\section{Ethics statement}

The Institutional Review Boards of Colorado State University and Poudre Valley Hospital approved this protocol (CSU protocol \#- 05-116H, PVH protocol \#- 07-874). Each volunteer was informed of the potential risks and written consent was obtained prior to enrolment. The study followed the guidelines set forth by the Declaration of Helsinki.

\section{Study overview}

A total of 15 morbidly obese gastric bypass patients, and 13 non-obese controls age 18-60 were recruited to participate through the Northern Colorado Surgical Associates (NCSA) of the Bariatric Center of the Rockies. Bypass patients were required to have a body mass index (BMI) $>40 \mathrm{~kg} / \mathrm{m}^{2}$, while controls were required to have a $B M I<30 \mathrm{~kg} / \mathrm{m}^{2}$. There were no other exclusion criteria allowing the subject population to be heterogeneous and, thus, differences in lipid profiles between groups are representative of the general clinical population. Subjects completed medical and exercise questionnaires prior to undergoing a venipuncture blood draw following a 12-hour fast. Blood was collected in vacutainer tubes containing EDTA, $0.5 \mathrm{~mL}$ were aliquoted for $\mathrm{HbA} 1 \mathrm{C}$ analysis, and the remainder was centrifuged (1200 g, $15 \mathrm{~min}, 4^{\circ} \mathrm{C}$ ) for collection of plasma which was stored at $-80^{\circ} \mathrm{C}$ until lipid extractions were performed.

\section{Subject characteristics}

Participant characteristics are shown in Table 1. We were unable to obtain body composition data because subject size exceeded the capacity of the available dual energy x-ray absorptiometry (DEXA) equipment. Height and weight were measured at the surgical center, while other demographic and health information was obtained through a health history questionnaire. None of the subjects were undergoing active weight reduction prior to surgery. Whole blood samples were sent to the University of Colorado Denver Clinical Translational Research Center for analysis of hemoglobin A1C (HbA1C) using a DCA Vantage analyzer (Siemens, Deerfield, IL).

\section{Lipid extraction}

Lipid extractions were performed using the methyl-tert butyl ether (MTBE) method described by Matyash et al. [20], with all steps performed in the Captair Pyramid 
Table 1 Subject characteristics

\begin{tabular}{lll}
\hline & Control & Obese \\
& $\mathbf{n = 1 3}$ & $\mathbf{n}=\mathbf{1 5}$ \\
& $\mathbf{( F = 1 1 ,} \mathbf{M}=\mathbf{2})$ & $\mathbf{( F = 1 0 ,} \mathbf{M}=\mathbf{5})$ \\
\hline Age (yr) & 42 & 44 \\
Height (in) & $65 \pm 3$ & $67 \pm 3$ \\
Weight (kg) & $71 \pm 14$ & $142 \pm 33^{*}$ \\
BMI (kg/m $\left.{ }^{2}\right)$ & $25.76 \pm 4.39$ & $49.87 \pm 11.27$ * \\
BMI min & 20 & 41 \\
BMI max & 31 & 87 \\
HbA1C (\% of total) & $5.15 \pm 0.10$ & $6.41 \pm 0.35 *$ \\
Current smokers & 2 & 1 \\
Former smokers & 4 & 11 \\
Statins & 2 & 1 \\
Anti-Hypertensives & 2 & 6 \\
Hypoglycemics & 0 & 4 \\
Anti-anxiety and anti-depressants & 4 & 5 \\
\hline
\end{tabular}

${ }^{*}=p<0.01$ compared to control. Data are presented as Mean \pm SE.

glove box (Erlab, Rowley, MA) under argon gas to prevent exposure to oxygen and subsequent oxidation of lipids. Briefly, $200 \mu \mathrm{L}$ plasma was added to $80 \mu \mathrm{L}$ of $\mathrm{MS}$ grade $\mathrm{H}_{2} \mathrm{O}$ in a glass tube with a Teflon lined cap, followed by addition of $1.5 \mathrm{~mL}$ of molecular grade methanol. The tubes were vortexed, $5 \mathrm{ml}$ of molecular grade MTBE was added, and then the samples were rocked for $1 \mathrm{hr}$ at room temperature. Following the incubation, $1.25 \mathrm{~mL}$ of MS grade $\mathrm{H}_{2} \mathrm{O}$ was added and the tubes were vortexed and centrifuged at $1,000 \times \mathrm{g}$ for $10 \mathrm{~min}$. The upper organic phase was collected, placed in another glass tube with a Teflon lined cap, and dried under nitrogen gas. Samples were re-suspended in $1 \mathrm{~mL}$ of LC-MS grade methanol and topped with high purity argon gas. Samples were stored for no longer than one week at $-20^{\circ} \mathrm{C}$ prior to chromatographic analysis. Samples were put in random order prior to lipid extraction, and again prior to mass spec analysis.

\section{Liquid chromatography/mass spectroscopy (LC/MS)}

Both the targeted and shotgun analysis were performed on lipid extracts, with lipids separated by reversed phase ultra performance liquid chromatography (UPLC) on an Acquity instrument (Waters, Milford MA). Solvent A consisted of an 89:5:5:1 mixture of water, isopropyl alcohol, acetonitrile and $500 \mathrm{mM}$ ammonium acetate respectively. Solvent B consisted of a 50:49:1 mixture of isopropyl alcohol, acetonitrile, and $500 \mathrm{mM}$ ammonium acetate, respectively. All solvents were LC-MS grade (Fisher, Optima LCMS). One microliter injections were loaded to a $1.0 \times 100 \mathrm{~mm}$ Acquity BEH C8 column held at $50^{\circ} \mathrm{C}$ with a $140 \mu \mathrm{L} / \mathrm{min}$ flow of $100 \%$ solvent A. Solvent A was held at $100 \%$ for $0.1 \mathrm{~min}$, followed by a linear gradient to $40 \%$ Solvent A over $0.9 \mathrm{~min}$. A second linear gradient to $100 \%$ Solvent B was achieved over $10 \mathrm{mi}$ nutes, followed by a $3 \mathrm{~min}$ hold at $100 \%$ Solvent B. The chromatographic system was returned to initial conditions via a 0.1 min linear gradient to $100 \%$ Solvent A, followed by a $5.9 \mathrm{~min}$ equilibration prior to the subsequent injection. Total run time was $20 \mathrm{~min}$. Eluate was introduced to a Q-ToF Micromass spectrometer (Waters/Micromass, Manchester, UK) via electrospray in either the positive or negative ionization mode. Capillary voltages were held at $3000 \mathrm{~V}$ and $2200 \mathrm{~V}$ in positive and negative ionization modes, respectively. In both modes the sample cone voltage was held at $30 \mathrm{~V}$, the collision energy was held at $7 \mathrm{~V}$, the source temperature was held at $130^{\circ} \mathrm{C}$, and the desolvation temperature was held at $300^{\circ} \mathrm{C}$. Data was acquired over the $100-1200 \mathrm{~m} / \mathrm{z}$ range in all analyses, at a rate of 2 scans/sec. The sample set was analyzed in independently randomized technical duplicate for each ionization mode. Both the UPLC and mass spectrometer were operated using MassLynx software version 4.1 (Waters, Milford, MA).

Peak detection and integration were performed using MarkerLynx software (Waters, Milford MA). Chromatographic peaks eluting between 0 and $14 \mathrm{~min}$ with $\mathrm{m} / \mathrm{z}$ values between 100 and 1200 were detected using a mass window of 0.07 Daltons and a retention time window of 0.1 min. Apex Track peak parameter settings were used, with peak width and baseline noise set for automatic calculation. A threshold of 10 counts/scan, a noise elimination level of 6 was implemented to minimize the detection of spectral noise, and the deisotope option was enabled to exclude isotopic peaks from the final data matrix of detected features and their intensities across all samples.

\section{OPLS modelling}

For the shotgun analysis, multivariate statistical analysis was performed using SIMCA- $\mathrm{P}^{+}$software (Umetrics Kinnelton, NJ). We used principal components analysis (PCA), and combined PLS and orthogonal single correction discriminant analysis (OPLS-DA) to analyze the LC/MS data. In short, PCA is an unbiased analysis of the total metabolite content (in our analysis the total plasma lipid profile) that detects variance among samples and provides sample clusters based on similarity of molecular profile. OPLS-DA is an extended form of partial least squares-discriminant analysis (PLS-DA) that, in addition to explaining overall differences between classes, separates predictive and non-predictive variation [21]. Data were modelled to visualize discrimination between the obese and control groups using the OPLS-DA model and scores plot of the first 
and second components. Goodness of fit was quantified by $R^{2} X$ and $R^{2} Y$, and predictability by $Q^{2} Y$.

\section{Lipid ion analysis}

A strict analysis and reduction to specific lipid ions of interest was performed using the models and scores plots from the OPLS-DA analysis, along with the raw LC/MS data. Factors in addition to obesity, including medication use in the control population, could influence and potentially weaken our model because of the heterogeneity of the subject population. However, lipids that emerge as significantly different between non-obese and obese despite the potential weakness of group heterogeneity, best represent the differences due to obesity in the general clinical population. First, a list was compiled of all the ions falling between 0.5 and 1 on the scores plots from both positive and negative mode because these ions were driving the difference between groups in the OPLS-DA model. An additional list was compiled containing all ions that were significantly different between groups based on t-tests comparing feature abundance between groups. All ions on the $t$-test list with a difference of less than 2 fold between obese and control groups were excluded, as were the features with abundances too low to be distinguished as an actual lipid ion, allowing us to narrow the list to approximately the most significantly different $10 \%$ of the ions. The remaining ion features on the $t$-test list were crossreferenced with the ion features on the scores plot list. A final list was compiled of ion features that were present on the scores plot list and final $t$-test list. These were the lipid ions that were both driving the difference between groups observed in the OPLS modelling, and that were statistically significantly different between groups. Ions on this list were further examined using tandem mass spectroscopy (MS/MS).

\section{Tandem mass spectroscopy and lipid identification}

Samples were analyzed by MS/MS as described above for the initial LC/MS analysis with the exception of selective mass filtering with the quadrupole for our ions of interest, and a collision energy of $40 \mathrm{~V}$. Fragmentation patterns were analyzed and cross referenced to Lipid Maps mass spectrometry peak prediction resources [22], and matched with published spectra where available for identification of lipids of interest. Standards were commercially available (Avanti Polar Lipids, Alabaster, AL) for three of the tentatively identified lipids of interest, 1-0-1'-(Z)-Octadecenyl-2-Arachidonoyl-snGlycero-3-Phosphoethanolamine, 1-Palmitoyl-2-Linoleoylsn-Glycero-3-Phosphocholine, and 1-octadecanoyl-2-(5Z, 8Z, 11Z, 14Z-eicosatetraenoyl)-sn-Glycero-3-Phospho(1'-myo-inositol))and we performed MS/MS on those available to confirm our tentative identifications. Following confirmation of our tentative identifications, we used the ether-linked phosphoethanolamine standard in cell culture treatments to determine if it induced pathological phenotypic changes in endothelial cells.

\section{Cell culture and lipid treatments}

Primary human coronary artery endothelial cells (HCAEC) (Lonza, Walkersville, MD) were grown in endothelial cell growth medium (EBM-2) containing 5\% FBS and manufacturer recommended supplemental growth factors, antibiotics, and antimycotics. All assays were performed on cells at $80-100 \%$ confluence, between passages 3 and 9. Lipid treatments included normal medium, vehicle controls, and the 1-0-1'-(Z)-Octadecenyl-2-Arachidonoyl-sn-Glycero3-Phosphoethanolamine (PE) standard. This PE standard was used for endothelial cell treatments as a representative ether-linked lipid because it was the ether-linked standard on our list that was commercially available. Treatment concentration for the PE standard was $25 \mu \mathrm{g} / \mathrm{mL}$, with all treatments performed for $4 \mathrm{hrs}$ and repeated a minimum of three times in duplicate or triplicate. HCAEC were also treated with L- $\alpha$-Phosphatidylcholine (PC) standard (Avanti Polar Lipids, Alabaster, AL), and PC standard that was oxidized by exposure to room air for 48 hrs as previously described [13], to confirm the effects of previously described oxidized PAPC in our model.

\section{Western blot analyses}

HCAEC were seeded in $65 \mathrm{~mm}$ polystyrene cell culture dishes and grown to at least $80 \%$ confluence prior to lipid treatment. Following treatment, cells were scraped in RIPA buffer (50 mM Tris, $0.15 \mathrm{M} \mathrm{NaCl}, 1 \% \mathrm{Na}$ deoxycholic acid, $1 \mathrm{mM}$ EGTA, 1\% NP40) containing protease and phosphatase inhibitors, and sonicated $3 \times 10 \mathrm{sec}$. Protein concentrations were determined using a BCA assay, and samples were diluted with the appropriate volume of Laemmli sample buffer for loading $25 \mu \mathrm{g}$ protein per well. Samples were separated on $7.5 \%$ polyacrylamide gels at $125 \mathrm{~V}$, and transferred to nitrocellulose membranes (BioRad, Hercules, CA) for $1 \mathrm{hr}$ at $50 \mathrm{~V}$. Membranes were blocked for $1 \mathrm{hr}$ in Superblock (Thermo Scientific, Rockford, IL) and then incubated with primary antibodies against VCAM (1:200) and ICAM (1:200) followed by the appropriate HRP-conjugated secondary antibodies. Membranes were developed by chemiluminescence using SuperSignal West Dura substrate (Thermo Scientific, Rockford, IL), with digital images obtained using the Biospectrum UVP system (Upland, CA). All signals were normalized to $\beta$-actin obtained from the same blot, and expressed as the percent of the normal medium control condition.

\section{ELISA analysis}

Monocyte chemoattractant protein-1 (MCP-1) in the cell culture medium was determined using a sandwich 
ELISA ( $\mathrm{R} \& \mathrm{D}$, Minneapolis, MN). The analysis was performed according to manufacturer instructions. The lower limit of detection was $31.2 \mathrm{pg} / \mathrm{mL}$ and average CV was $2.2 \%$.

\section{Statistical analysis}

Modelling and analysis of LC/MS data are described above. Comparison of ion abundance between obese and control groups, and lipid treatment effects on HCAEC were performed using unpaired t-tests. Significance was set $a$ priori at $\mathrm{p} \leq 0.05$.

\section{Results}

\section{Subject characteristics}

There was no difference in average age between groups, but average BMI was significantly higher $(\mathrm{p}<0.01)$ in the morbidly obese group $\left(49.87 \pm 11.27 \mathrm{~kg} / \mathrm{m}^{2}\right)$ compared to control $\left(25.76 \pm 4.39 \mathrm{~kg} / \mathrm{m}^{2}\right)$ and $\mathrm{HbA1C}$ was significantly higher $(\mathrm{p}<0.01)$ in the morbidly obese group $(6.41 \pm$ $0.35 \%$ of total) compared to control $(5.15 \pm 0.10 \%$ of total) (Table 1).

\section{Global lipidomic analysis}

For both the shotgun and targeted analysis we analysed lipid extracts from control and obese subjects using LC/MS. We then began the analysis for the shotgun approach with the principle component analysis (PCA). PCA models (Figure 1) for positive and negative mode poorly classified the control and obese groups, which was not unexpected given the heterogeneity of the groups. The PCA model eliminated outliers prior to the biased OPLSDA analysis. OPLS-DA modelling was performed on the remaining control and obese lipid profile data obtained in both positive and negative mode. The positive mode model and a representative scores plot are shown in Figure 2A, the negative mode model and a representative scores plot

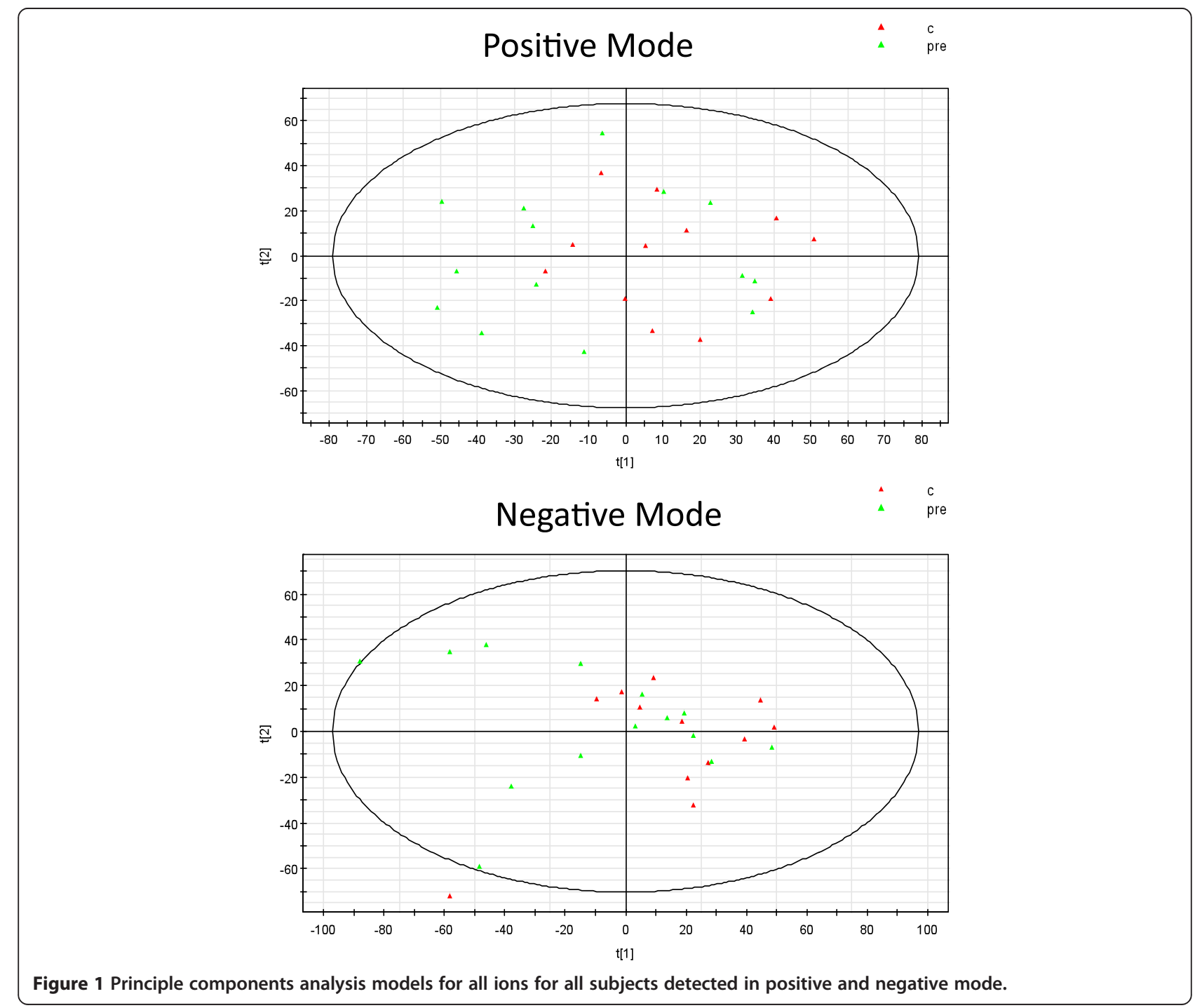




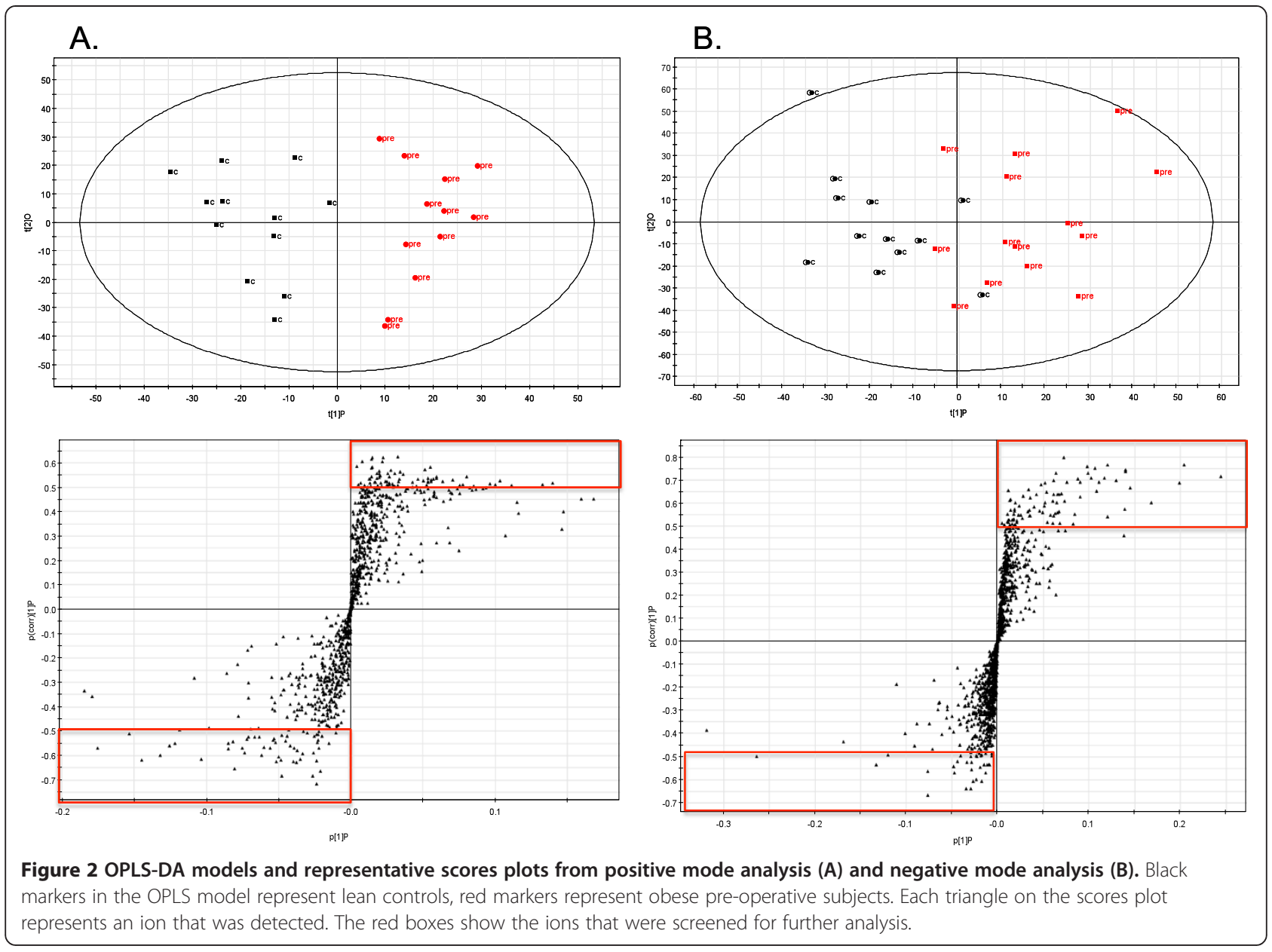

are shown in Figure $2 B \cdot R^{2} X, R^{2} Y$, and $Q^{2} Y$ of the positive mode model were $0.298,0.839$, and 0.259 respectively, while $R^{2} X, R^{2} Y$, and $Q^{2} Y$ of the negative mode model were $0.49,0.658$, and 0.216 respectively. Our values are similar to analyses in other heterogeneous diseased human populations [23]. Based on the OPLS-DA models and statistical analysis as described above, a list of 43 lipid ions of interest that were significantly different between groups and driving the group separation in the OPLS-DA model was compiled for further examination (Additional file 1: Table S1), 26 of those were greater in obese subjects.

\section{Lipid ion of interest identification}

MS/MS analysis was performed on all lipid ions of interest. Following MS/MS analysis, screening of spectra, comparison of fragments in Lipid Maps, comparison to published spectra, and elimination of ions that were unidentifiable or became negligible, 22 ions of interest were tentatively identified. Numerous tentatively identified ions were present as multiple adducts, Table 2 shows the final list of ions of interest that were greater in obese subjects and of interest for further study. We discovered that multiple ether-linked phospholipids were significantly higher in the obese group compared to control and were driving group separation in the OPLS-DA model.

To confirm our tentative ion identifications, where available we compared previously published spectra of our tentatively identified ions to our spectra. Figure 3 shows a representative MS/MS spectra of $\mathrm{PE}(\mathrm{P}-18: 0 / 20: 4$ $(5 Z, 8 Z, 11 Z, 14 Z))$ an ether-linked phosphatidylethanolamine (PE), in one of our samples, that was identified in negative mode, and a previously published spectra of the same ion confirming our tentative identification of that ion. Published spectra for this PE can also be found in Goodenowe et al. showing the same fragmentation pattern [24]. We also purchased the standards that were available from our list to analyze via MS/MS and compared the spectra of our unknown ions to the spectra of the standards. Spectra from the standards that were analysed matched the spectra of our unknowns confirming the identity of those ions. Figure 4 shows the chromatography and MS/MS spectra of PI(18:0/20:4(5Z,8Z,11Z,14Z)) from the purchased standard (top chromatogram and 
Table 2 Identifications of ions from MS/MS analysis

\begin{tabular}{|c|c|c|c|c|}
\hline $\begin{array}{r}\text { Ion of interest from } \\
\text { original LC/MS analysis }\end{array}$ & Tentative ID based on MS/MS analysis & P-value & Ave (c) & Ave (pre) \\
\hline 750.5399 & PE(P-18:0/20:4(5Z,8Z,11Z,14Z)) & 0.016 & 8.25 & 13.73 \\
\hline 838.5599 & PE(18:2(9Z,12Z)/20:2(11Z,14Z)) & 0.039 & 17.67 & 27.82 \\
\hline 829.57 & $P C(16: 0 / 18: 2(9 Z, 12 Z))$ & 0.022 & 0.01 & 0.18 \\
\hline 802.5599 & $P C(0-16: 0 / 18: 2(9 Z, 12 Z))$ & 0.019 & 49.22 & 64.29 \\
\hline 816.57 & $P C(O-16: 0 / 18: 2(9 Z, 12 Z))$ & 0.031 & 161.81 & 208.10 \\
\hline 830.59 & $P C(O-18: 0 / 18: 2(9 Z, 12 Z))$ & 0.003 & 32.48 & 45.40 \\
\hline 844.5999 & $P C(0-18: 0 / 18: 2(9 Z, 12 Z))$ & 0.005 & 94.68 & 130.23 \\
\hline 832.5999 & PC(O-18:0/18:1(9Z)) & 0.005 & 8.85 & 14.46 \\
\hline 868.5999 & $P C(O-18: 0 / 20: 4(5 Z, 8 Z, 11 Z, 14 Z))$ & 0.010 & 41.54 & 63.87 \\
\hline 870.6199 & PC(O-18:0/20:3(8Z,11Z,14Z)) & 0.028 & 30.86 & 44.71 \\
\hline 885.5499 & $\mathrm{PI}(18: 0 / 20: 4(5 Z, 8 Z, 11 Z, 14 Z))$ & 0.011 & 45.66 & 62.11 \\
\hline
\end{tabular}

$\mathrm{p}$-values were calculated using an unpaired $t$-test comparison of group relative abundance means for each ion from the original run. $\mathrm{c}=\mathrm{control}$, pre $=$ obese pre-surgical. Ave (c) and (pre) values are average relative abundance in arbitrary units of each ion for each group from the original run.

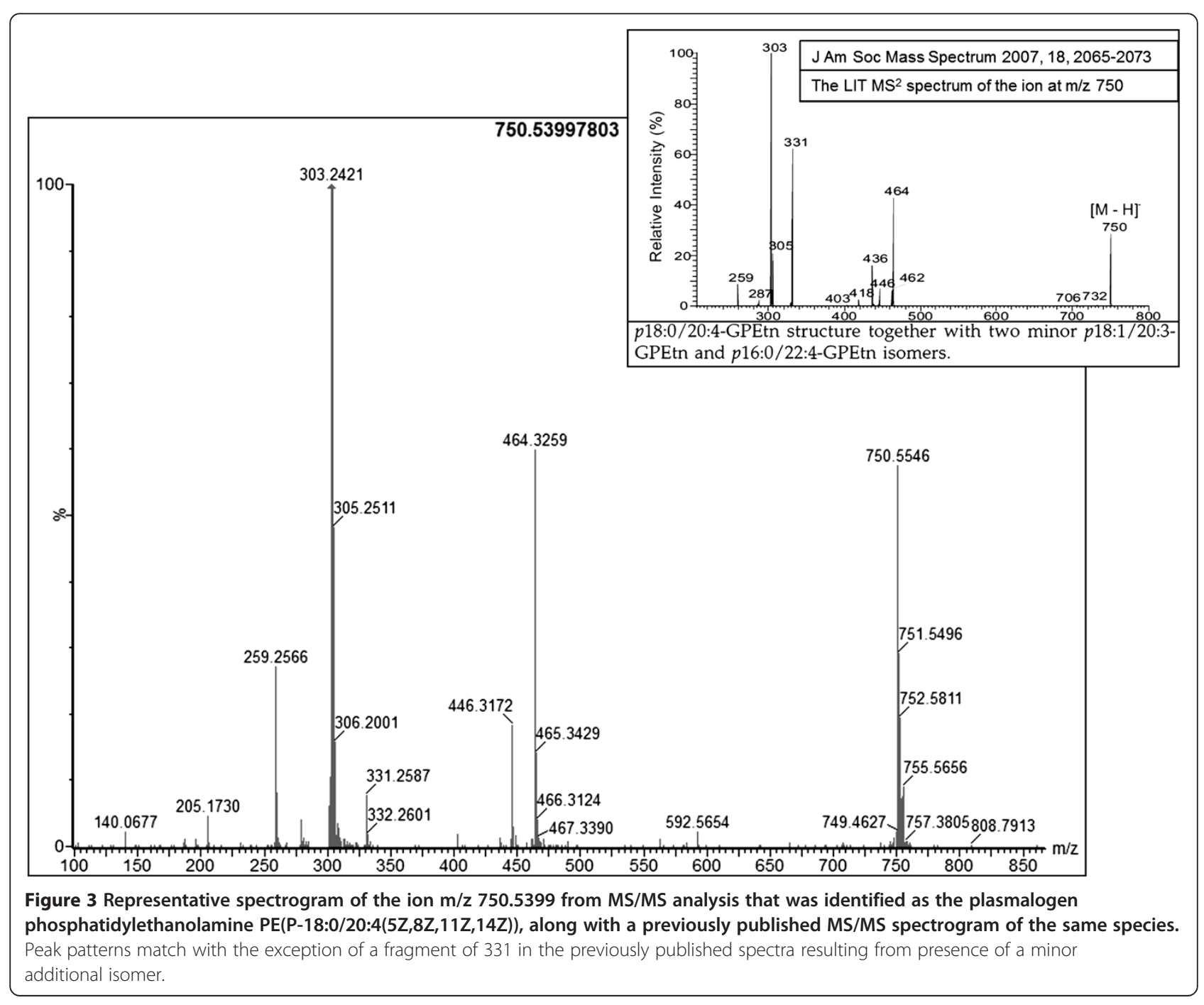




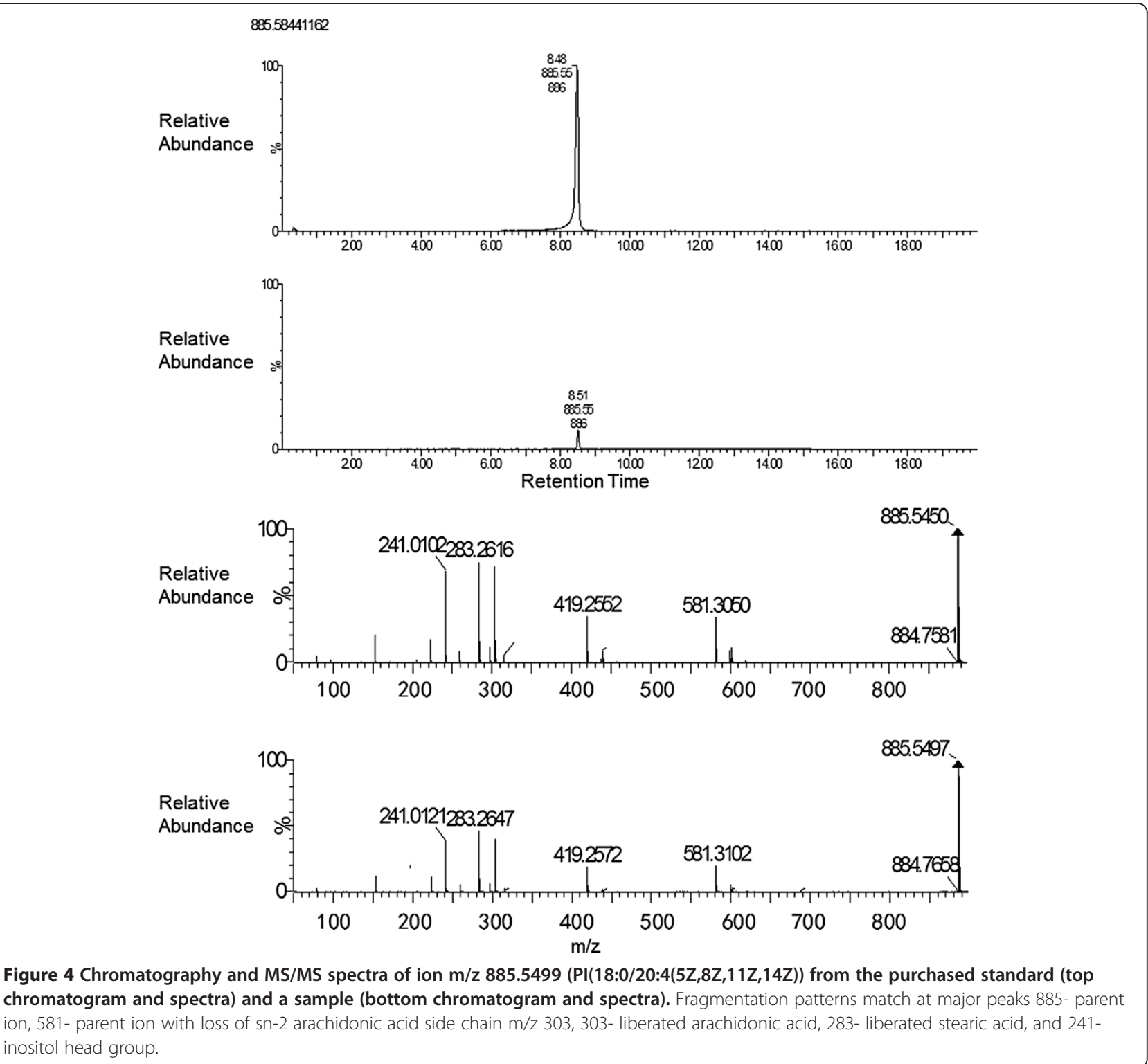

spectra) and our sample (bottom chromatogram and spectra). Retention times match, and major identifying peaks include 885 (parent ion) 303 (20-4 arachidonic acid) 283 (18-0 steric acid), and 241 (inositol head group). Because the exceptional finding from the shotgun analysis was the emergence of the group of ether-linked lipids, we next sought to examine what potential effects ether-linked lipids may have on endothelial cells, to obtain insight into the relationship between obesity associated dyslipidemia and vascular disease.

\section{Endothelial cell response to ether-linked} phosphatidylethanolamine and oxidized PAPC

To determine the effect of our identified ether linked lipids on endothelial cells, we used PE-(P-18:0/20:4
$(5 \mathrm{Z}, 8 \mathrm{Z}, 11 \mathrm{Z}, 14 \mathrm{Z}))(\mathrm{p}-\mathrm{PE})$, the only commercially available ether-linked lipid on our final list, to treat HCAEC. p-PE induced increases in the cell adhesions molecules ICAM (158\% of control, $\mathrm{p}=0.028)$ and VCAM (144\% of control, $\mathrm{p}=0.038$ ) (Figure 5). Cell culture medium was collected for analysis of the secreted inflammatory mediator MCP-1. MCP-1 did not differ in medium collected from cells incubated in normal medium, and those that were exposed to p-PE (Figure 6). Because previous studies have described induction of ICAM and VCAM by oxidized phospholipids, we also used oxidized phosphatidylcholine (ox-PC) to treat HCAEC. Ox-PC also induced increases in ICAM $(180 \%$ of control, $\mathrm{p}=0.038)$ and VCAM (169\% of control, $\mathrm{p}=0.051)$, while there was no effect of un-oxidized PC. 

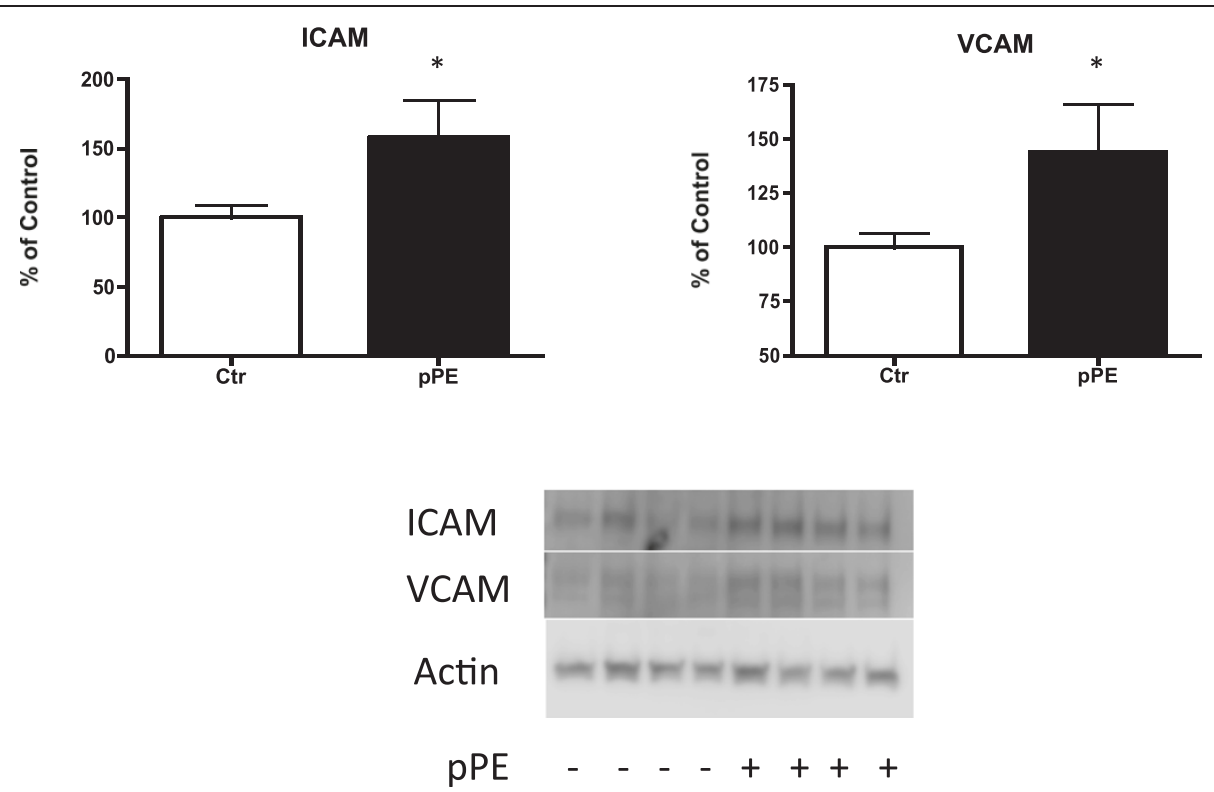

Figure 5 Effects of lipid treatment on HCAEC cell adhesion molecule protein expression. pPE increased ICAM protein (158\% of control, $p=0.028)$ and VCAM protein ( $144 \%$ of control, $p=0.038)$ in HCAEC. Data are presented as Mean \pm SE.

\section{Targeted analysis of oxidized phosphatidylcholine}

Because previous in vitro data indicate that ox-PC products cause pathological phenotypic changes in endothelial cells, we targeted PC and ox-PC in our LC/MS data from morbidly obese and control plasma using a targeted approach. We scanned all ions in the positive mode analysis for any that could be PC, PGPC, POVPC, or PEIPC based on $\mathrm{m} / \mathrm{z}$ and abundance. While none of the best potential matches for these lipids were between 0.5 and 1 in our OPLS-DA models, one potential PC match appeared to be significantly higher in obese

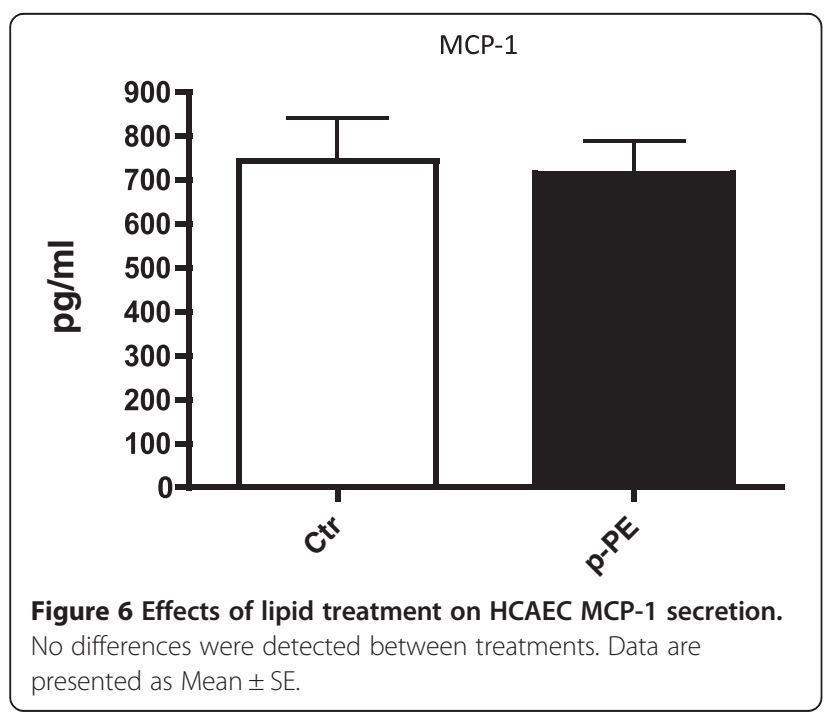

subjects compared to control $(\mathrm{p}=0.040)$, though the other potential match had a much higher relative abundance (as would be expected for PC because it is the most abundant phospholipid in cells) and was not significantly different. Of the best matches for the ox-PC species, only those for PGPC were close to significantly elevated in the obese compared to control $(p=0.052$, and 0.055), while the best potential matches for PEIPC were not significant $(p=0.707$, and 0.661$)$ and no ions that could represent POVPC were detected (Table 3).

\section{Discussion}

Lipidomics often functions as a hypothesis generating technique where biomarkers of interest emerge from large data sets indicating further analysis of physiological roles of identified features. Because obesity is a major independent risk factor for coronary artery disease, and a major component of that risk is dyslipidemia, we sought to characterize the lipid profiles from morbidly obese human subjects compared to control. We combined in vivo and in vitro methods to examine the effects of lipids that were identified and elevated in obese subjects on pathophysiology of endothelial cells. The major findings of this study were that a group of ether-linked lipids was elevated in plasma from morbidly obese subjects compared to control, and PE-(P-18:0/20:4(5Z,8Z,11Z,14Z)) increased cell adhesion molecule protein but not the inflammatory mediator MCP-1 in endothelial cells. In addition, PAPC, PGPC, POVPC, and PEIPC were not elevated in our obese group compared to control. 
Table 3 Oxidized PAPC products from positive mode analysis

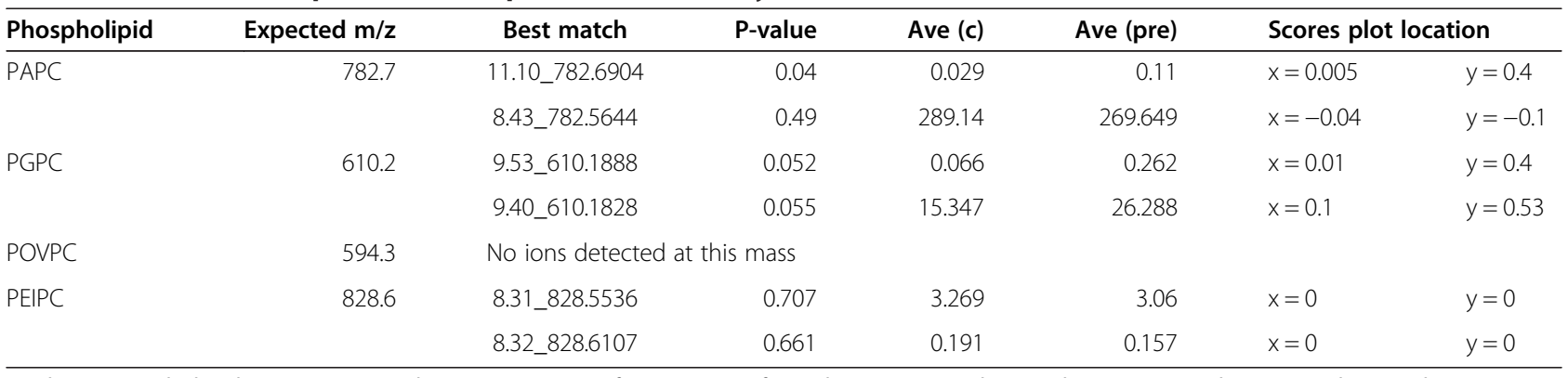

p-values were calculated using an unpaired $t$-test comparison of group means for each ion. $c=$ control, pre $=$ obese pre-surgical. Ave (c) and (pre) values are average relative abundance in arbitrary units of each ion for each group. Scores plot locations represent the coordinates of the location of each ion on the positive mode scores plot shown in Figure 3.

\section{Ether-linked lipids}

There was a significant difference in the lipid profiles between our control and obese subjects. Following our conservative and strict approach to narrowing down the total lipid profile to a small list of lipids of interest, a group of ether-linked phospholipids that were elevated in obese subject plasma compared to control that were driving the separation of groups in our model emerged. Ether-linked lipids are characterized by an ether linkage as opposed to an ester linkage at one or both fatty acid side chains, with the subclass plasmalogens having a vinyl ether linkage. Standard ether-linked lipids as well as plasmalogens both appeared on our list, and the ether-linked phosphatidylethanolamine used in our in vitro experiments was a plasmalogen.

Ether-linked lipids have been characterized in macrophages, lens cells, adipose, liver, and serum [25-27]. In the context of metabolic disease, reports detailing etherlinked lipid presence and alteration vary. A recent lipidomic analysis on serum, liver, subcutaneous adipose, and intraabdominal adipose from a group of obese subjects was showed that ether-linked PEs were higher in liver compared to both adipose depots, but ether-linked PCs were higher in adipose depots [26]. In this analysis ester PCs were more abundant in all tissues compared to ether-linked, but ether-linked PEs were more abundant than esters in all tissues. Graesssler et al. found a significant increase in multiple PE plasmalogens in individuals with $\mathrm{BMI}>27.5$, but diminished ether-linked lipids in individuals with increasing HOMA-IR [19]. A comparison of obese and non-obese twins found that more ether-linked phospholipids were diminished in the obese twin compared to the lean than were elevated [28]. Other recent work profiling lipid composition of LDL found that plasmalogen PEs are decreased in metabolic syndrome patients compared to lean, as well as in diabetics compared to lean, and the proportion of plasmalogen PE negatively correlates with waist circumference [29]. Conversely, it has also been reported that plasmalogen presence is greater in hypercholesterolemic subjects [30], and that the ratio of plasmalogen PE to ester PE was increased in a population of diabetic patients without cardiovascular complications compared to controls [31]. In the present study we did not have enough subjects to stratify results based on diabetes in our obese group. Collectively, the work of others as well as the current investigation support further examination of why ether-linked phospholipids vary between lean control and obese subjects and what the role of ether-linked lipids in metabolic disease may be. It may be that the effects and presence of individual ether-linked and plasmalogen PCs and PEs varies depending on individual disease severity and milieu.

\section{Physiological roles of ether-linked lipids}

Ether-linked phospholipids are present in most cell membranes and studies of their function have ranged from signalling in brain cells to antioxidant properties [32-35]. There is a lack of consensus about whether ether-linked lipids are pro-or anti-oxidant and inflammatory. Ether-linked lipids, such as those identified in the current study, are more susceptible to oxidation compared to ester-linked lipids [34,35]. Ether-linked plasmalogens in particular are preferentially oxidized over ester-linked phospholipids, possibly due to structural differences leaving the polyunsaturated sn-2 side chains more exposed [36], and lower bond dissociation energies of ether linkages [33]. Decreased plasmalogens in LDL from patients with the metabolic syndrome and diabetes has been suggested as an indicator of oxidative stress [29]. In the current study it may be that the observed ether-linked lipids are elevated as an antioxidant response to increasing oxidative stress with obesity, and as noted earlier reports vary in regard to increases and decreases in ether-linked lipids based on various symptoms of the metabolic syndrome. Whether ether-linked lipids function as ROS scavengers or are simply more susceptible to oxidation remains to be seen. However if 
ether-linked lipids are scavengers and are preferentially synthesized in response to a pro-oxidant environment or altered membrane fluidity, this may be of interest in reference to obese and diabetic populations where increased oxidative stress is often a problem.

Ether-linked lipids may be pro-inflammatory and can serve as arachidonic acid reservoirs. Ether-linked lipids, particularly plasmalogens have a high proportion of arachidonic acid at the sn- 2 position, and are involved in membrane remodeling as well as intracellular arachidonic acid metabolism $[15,16]$. Arachidonic acid released from the sn-2 position of ether and ester lipids can be enzymatically oxidized to form prostaglandins, prostacyclin, thromboxanes, and leukotrienes, all of which are pro-inflammatory $[37,38]$. Ether-linked lipids and oxidation products of ether-linked lipids include proinflammatory lysophospholipids that are platelet activating factor (PAF) precursors, and lysophospholipids independently can be pro-inflammatory and pathological. Lysophosphocholines (LPCs) have been shown to increase plasminogen activator inhibitor-1, a potent prothrombotic and proatherogenic protein [39]. In addition, LPCs can induce endothelial cell expression of cell adhesion molecules, an important step in endothelial cell activation and atherosclerotic progression $[40,41]$.

Ether-linked lipids with arachidonic acid at the sn-2 position serve as PAF synthesis precursors. PAF is an ether-linked species with a choline head group, an etherlinked fatty acid at the sn-1 position and an acetyl group at the sn-2 position that is a potent stimulator of the platelet coagulation cascade, thrombosis, and inflammation. PAF has been implicated in atherogenesis because it activates and recruits inflammatory cells, and in advanced plaque rupture because it initiates the coagulation cascade in platelets (reviewed in [42]). What is unknown is whether PAF formation only occurs in response to external stimuli, or if formation of PAF can be driven by excess substrate availability. The ether-linked phospholipids, including those identified in our analysis with arachidonic acid at the sn- 2 position and choline head groups, may serve as substrate for PAF formation and thus be characterized as pro-inflammatory.

\section{Ether-linked phospholipids and HCAEC}

To address whether ether-linked lipids can induce phenotypic changes in endothelial cells, we performed in vitro experiments using a commercially available ether-linked PE, PE-(P-18:0/20:4(5Z,8Z,11Z,14Z)), that corresponded to a species we identified as elevated in obese subjects. This PE stimulated cell adhesion molecule expression in human coronary artery endothelial cells a key step in atherogenesis. The increases we observed in cell adhesion molecule expression were similar in magnitude to those observed in endothelial cells following oxidized PAPC treatment [19].

We cannot conclude based on these data that the effects of this plasmalogen phosphatidylethanolamine are representative of other ether-linked lipids, including those ether-linked phosphatidylcholines that were also identified during our analysis. However, our data suggest that this lipid may induce changes in endothelial cells indicative of vascular disease, and support further analysis of the role of ether linked lipids in the vascular endothelium.

\section{Oxidized phospholipids}

The finding that phosphatidylcholine oxidation products that have been shown in vitro to induce disease related phenotypic changes in endothelial cells were not elevated in our obese subjects was unexpected. Because oxidized phosphatidylcholine products have been found in modified LDL and atherosclerotic lesions from high fat fed rabbits [6] and oxidized LDL has been found in lesions isolated from human vessels [43], we hypothesized they would be elevated in morbidly obese subjects compared to control. Our findings do not discredit the data indicating detrimental effects of oxidized PAPC on endothelial cells, however oxidized PAPC products did not drive the separation between control and obese groups in our model. Our data support the importance of examining other classes of lipids and presence of oxidized phospholipids in human plasma and LDL.

\section{Lipidomic and metabolomic analyses}

Each lipidomic and metabolomic analysis is performed using unique methods and parameters, and generates unique results contributing to global understanding of group differences and metabolism. Lipidomics has been used recently to compare tissues in subjects with the metabolic syndrome and diabetes, overweight and obese subjects, and gastric bypass patients among others [26,44-46]. While lipidomic and metabolomic studies don't necessarily address mechanistic questions, information obtained using these techniques provides information on global differences between groups and often drives hypothesis development for mechanistic questions. As such, our analysis revealed a group of etherlinked lipids that are elevated in morbidly obese humans. Further, these findings translated to examination of the pathophysiological roles for this class of lipids, where PE-(P-18:0/20:4(5Z,8Z,11Z,14Z)) induces cell adhesion molecule expression in coronary artery endothelial cells. Limitations in our analysis include a small sample size, and while all preparation and analysis of samples following collection was performed under inert gas, we did not use an internal antioxidant. The complete class of etherlinked lipids found in biological samples is very large 
and the ether-linked lipids on our list represent a very small percentage, therefore its possible that our data are not generalizable to ether-linked lipids as a class. Regardless, the emergence of the ether-linked lipids of interest is strongly supported. Data in our lipidomic analysis show elevated ether-linked lipids in morbidly obese subjects compared to control. Given the potential pathophysiological roles of ether-linked lipids, our data support further investigation of the role this class may play in obesity associated vascular and metabolic disease.

\section{Additional file}

Additional file 1: Table S1. lons of interest identified in LC/MS analysis that were selected for MS/MS. p-value was calculated using an unpaired $t$-test comparison of group means for each ion.

\section{Competing interests}

The authors have no competing interests to disclose.

\section{Authors' contributions}

ELD contributed to study design, data collection and analysis, and manuscript preparation. SMP contributed to subject recruitment and data collection. MSH contributed to study design and manuscript preparation and analysis. KLH and BFM contributed to study design, funding, and manuscript preparation and analysis. All authors read and approved the final manuscript.

\section{Authors' information}

Karyn L Hamilton and Benjamin F Miller Co-principle investigators.

\section{Acknowledgements}

The authors would like to thank Shanyn Willis, Leora Garcia, Dr. Melinda Frye, Dr. Chris Bell, and Dr. Corey Broeckling from CSU, as well as Drs. Robert Quaid, Michael Roller, James Dickinson, and all nurses and staff at Northern Colorado Surgical Associates and the Bariatric Center of the Rockies for their contributions and assistance.

\section{Funding}

CSU Proteomics and Metabolomics Facility Academic Enrichment Program.

\section{Author details}

'Department of Health and Exercise Science, Colorado State University, Fort Collins, CO 8052, USA. ${ }^{2}$ Bariatric Center of the Rockies, Fort Collins, CO, USA. ${ }^{3}$ The Liggins Institute, University of Auckland, 85 Park Rd Grafton, Auckland NZ 1142, New Zealand.

Received: 12 February 2013 Accepted: 9 May 2013

Published: 14 May 2013

\section{References}

1. Dandona P, Aljada A, Chaudhuri A, Mohanty P, Garg R: Metabolic syndrome. Circulation 2005, 111:1448-1454.

2. Negre-Salvayre A, Auge N, Ayala V, Basaga H, Boada J, Brenke R, Chapple S, Cohen G, Feher J, Grune T, et al: Pathological aspects of lipid peroxidation. Free Radic Res 2010, 44:1125-1171.

3. Holvoet P, Lee D-H, Steffes M, Gross M, Jacobs DR: Association between circulating oxidized Low-density lipoprotein and incidence of the metabolic syndrome. JAMA 2008, 299:2287-2293.

4. Njajou OT, Kanaya AM, Holvoet P, Connelly S, Strotmeyer ES, Harris TB, Cummings SR, Hsueh W-C: Association between oxidized LDL, obesity and type 2 diabetes in a population-based cohort, the health, aging and body composition study. Diabetes Metab Res Rev 2009, 25:733-739.

5. Unger RH, Clark GO, Scherer PE, Orci L: Lipid homeostasis, lipotoxicity and the metabolic syndrome. Biochimica et Biophysica Acta (BBA) Molecular and Cell Biology of Lipids 2010, 1801:209-214.
6. Subbanagounder G, Leitinger N, Schwenke DC, Wong JW, Lee H, Rizza C, Watson AD, Faull KF, Fogelman AM, Berliner JA: Determinants of bioactivity of oxidized phospholipids. Specific oxidized fatty acyl groups at the sn-2 position. Arterioscler Thromb Vasc Biol 2000, 20:2248-2254.

7. Berliner JA, Gharavi NM: Endothelial cell regulation by phospholipid oxidation products. Free Radic Biol Med 2008, 45:119-123.

8. Berliner JA, Leitinger $\mathrm{N}$, Tsimikas $\mathrm{S}$ : The role of oxidized phospholipids in atherosclerosis. J Lipid Res 2009, 50(Suppl):S207-S212.

9. Berliner JA, Watson AD: A role for oxidized phospholipids in atherosclerosis. N Engl J Med 2005, 353:9-11.

10. Bochkov VN: Inflammatory profile of oxidized phospholipids. Thromb Haemost 2007, 97:348-354

11. Fu P, Birukov KG: Oxidized phospholipids in control of inflammation and endothelial barrier. Trans/ Res 2009, 153:166-176.

12. Leitinger $\mathrm{N}$ : Oxidized phospholipids as triggers of inflammation in atherosclerosis. Mol Nutr Food Res 2005, 49:1063-1071.

13. Watson AD, Leitinger N, Navab M, Faull KF, Horkko S, Witztum JL, Palinski W, Schwenke D, Salomon RG, Sha W, et al: Structural identification by mass spectrometry of oxidized phospholipids in minimally oxidized low density lipoprotein that induce monocyte/endothelial interactions and evidence for their presence in vivo. J Biol Chem 1997, 272:13597-13607.

14. Catala A: Lipid peroxidation of membrane phospholipids generates hydroxy-alkenals and oxidized phospholipids active in physiological and/or pathological conditions. Chem Phys Lipids 2009, 157:1-11.

15. Chilton FH, Murphy RC: Remodeling of arachidonate-containing phosphoglycerides within the human neutrophil. J Biol Chem 1986, 261:7771-7777.

16. Tamby JP, Reinaud P, Charpigny G: Preferential esterification of arachidonic acid into ethanolamine phospholipids in epithelial cells from ovine endometrium. J Reprod Fertil 1996, 107:23-30.

17. Wallner S, Schmitz G: Plasmalogens the neglected regulatory and scavenging lipid species. Chem Phys Lipids 2011, 164:573-589.

18. Colas R, Sassolas A, Guichardant M, Cugnet-Anceau C, Moret M, Moulin P, Lagarde M, Calzada C: LDL from obese patients with the metabolic syndrome show increased lipid peroxidation and activate platelets. Diabetologia 2011, 54:2931-2940.

19. Graessler J, Schwudke D, Schwarz PE, Herzog R, Shevchenko A, Bornstein SR: Top-down lipidomics reveals ether lipid deficiency in blood plasma of hypertensive patients. PLoS One 2009, 4:e6261.

20. Matyash V, Liebisch G, Kurzchalia TV, Shevchenko A, Schwudke D: Lipid extraction by methyl-tert-butyl ether for high-throughput lipidomics. $J$ Lipid Res 2008, 49:1137-1146.

21. Bylesj M, Holmes E, Trygg J, Rantalainen M, Cloarec O, Nicholson JK: OPLS discriminant analysis: combining the strengths of PLS-DA and SIMCA classification. J Chemometr 2006, 20:341-351.

22. Fahy ESM, Cotter D, Subramaniam S: LIPID MAPS online tools for lipid research. LIPID MAPS Online Tools 2007, 35:W606-W612

23. Castro-Perez JM, Kamphorst J, DeGroot J, Lafeber F, Goshawk J, Yu K, Shockcor JP Vreeken R, Hankemeier T: Comprehensive LC-MS E lipidomic analysis using a shotgun approach and its application to biomarker detection and identification in osteoarthritis patients. J Proteome Res 2010, 9:2377-2389.

24. Goodenowe DB, Cook LL, Liu J, Lu Y, Jayasinghe DA, Ahiahonu PW, Heath $D$, Yamazaki Y, Flax J, Krenitsky KF, et al: Peripheral ethanolamine plasmalogen deficiency: a logical causative factor in Alzheimer's disease and dementia. J Lipid Res 2007, 48:2485-2498.

25. Deeley JM, Thomas MC, Truscott RJ, Mitchell TW, Blanksby SJ: Identification of abundant alkyl ether glycerophospholipids in the human lens by tandem mass spectrometry techniques. Anal Chem 2009, 81:1920-1930.

26. Kotronen A, Seppanen-Laakso T, Westerbacka J, Kiviluoto T, Arola J, Ruskeepaa AL, Yki-Jarvinen H, Oresic M: Comparison of lipid and fatty acid composition of the liver, subcutaneous and intra-abdominal adipose tissue, and serum. Obesity (Silver Spring) 2010, 18:937-944.

27. Ivanova PT, Milne SB, Brown HA: Identification of atypical ether-linked glycerophospholipid species in macrophages by mass spectrometry. $J$ Lipid Res 2010, 51:1581-1590.

28. Pietilainen $\mathrm{KH}$, Sysi-Aho M, Rissanen A, Seppanen-Laakso T, Yki-Jarvinen $H$ Kaprio J, Oresic M: Acquired obesity is associated with changes in the serum lipidomic profile independent of genetic effects-a monozygotic twin study. PLoS One 2007, 2:e218.

29. Colas R, Sassolas A, Guichardant M, Cugnet-Anceau C, Moret M, Moulin P, Lagarde M, Calzada C: LDL from obese patients with the metabolic 
syndrome show increased lipid peroxidation and activate platelets. Diabetologia 2011, 54:2931-2940.

30. Brosche T: Plasmalogen levels in serum from patients with impaired carbohydrate or lipid metabolism and in elderly subjects with normal metabolic values. Arch Gerontol Geriatr 2001, 32:283-294.

31. Goto I, Antoku Y, Hayakawa T: Plasma plasmalogens of the phosphoethanolamine type and angiopathy in diabetic patients. Klin Wochenschr 1990, 68:49.

32. Han $X$, Holtzman DM, McKeel DW Jr: Plasmalogen deficiency in early Alzheimer's disease subjects and in animal models: molecular characterization using electrospray ionization mass spectrometry. J Neurochem 2001, 77:1168-1180

33. Lessig J, Fuchs B: HOCl-mediated glycerophosphocholine and glycerophosphoethanolamine generation from plasmalogens in phospholipid mixtures. Lipids 2010, 45:37-51.

34. Engelmann B, Brautigam C, Thiery J: Plasmalogen phospholipids as potential protectors against lipid peroxidation of low density lipoproteins. Biochem Biophys Res Commun 1994, 204:1235-1242.

35. Vance JE: Lipoproteins secreted by cultured rat hepatocytes contain the antioxidant 1-alk-1-enyl-2-acylglycerophosphoethanolamine. Biochim Biophys Acta 1990, 1045:128-134.

36. Khaselev N, Murphy RC: Susceptibility of plasmenyl glycerophosphoethanolamine lipids containing arachidonate to oxidative degradation. Free Radic Biol Med 1999, 26:275-284.

37. Nanda BL, Nataraju A, Rajesh R, Rangappa KS, Shekar MA, Vishwanath BS: PLA2 Mediated arachidonate free radicals: PLA2 inhibition and neutralization of free radicals by anti-oxidants-a new role as antiinflammatory molecule. Curr Top Med Chem 2007, 7:765-777.

38. Ricciotti E, FitzGerald GA: Prostaglandins and inflammation. Arterioscler Thromb Vasc Biol 2011, 31:986-1000.

39. Kuniyasu A, Tokunaga M, Yamamoto T, Inoue S, Obama K, Kawahara K, Nakayama H: Oxidized LDL and lysophosphatidylcholine stimulate plasminogen activator inhibitor-1 expression through reactive oxygen species generation and ERK1/2 activation in 3T3-L1 adipocytes. Biochim Biophys Acta 2011, 1811:153-162.

40. Kume N, Cybulsky MI, Gimbrone MA Jr: Lysophosphatidylcholine, a component of atherogenic lipoproteins, induces mononuclear leukocyte adhesion molecules in cultured human and rabbit arterial endothelial cells. J Clin Invest 1992, 90:1138-1144.

41. Lee H, Lin Cl, Liao JJ, Lee YW, Yang HY, Lee CY, Hsu HY, Wu HL: Lysophospholipids increase ICAM-1 expression in HUVEC through a Giand NF-kappaB-dependent mechanism. Am J Physiol Cell Physiol 2004, 287:C1657-C1666.

42. Ninio E: Phospholipid mediators in the vessel wall: involvement in atherosclerosis. Curr Opin Clin Nutr Metab Care 2005, 8:123-131.

43. Vickers KC, Maguire CT, Wolfert R, Burns AR, Reardon M, Geis R, Holvoet $P$, Morrisett JD: Relationship of lipoprotein-associated phospholipase A2 and oxidized low-density lipoprotein in carotid atherosclerosis. J Lipid Res 2009, 50:1735-1743

44. Wang C, Kong H, Guan Y, Yang J, Gu J, Yang S, Xu G: Plasma phospholipid metabolic profiling and biomarkers of type 2 diabetes mellitus based on high-performance liquid chromatography/electrospray mass spectrometry and multivariate statistical analysis. Anal Chem 2005, 77:4108-4116.

45. Kim JY, Park JY, Kim OY, Ham BM, Kim HJ, Kwon DY, Jang Y, Lee JH: Metabolic profiling of plasma in overweight/obese and lean men using ultra performance liquid chromatography and Q-TOF mass spectrometry (UPLC-Q-TOF MS). J Proteome Res 2010, 9:4368-4375.

46. Mutch DM, Fuhrmann JC, Rein D, Wiemer JC, Bouillot JL, Poitou C, Clement $\mathrm{K}$ : Metabolite profiling identifies candidate markers reflecting the clinical adaptations associated with roux-en-Y gastric bypass surgery. PLoS One 2009, 4:e7905.

doi:10.1186/1758-5996-5-24

Cite this article as: Donovan et al: Lipidomic analysis of human plasma reveals ether-linked lipids that are elevated in morbidly obese humans compared to lean. Diabetology \& Metabolic Syndrome 2013 5:24.

\section{Submit your next manuscript to BioMed Central and take full advantage of:}

- Convenient online submission

- Thorough peer review

- No space constraints or color figure charges

- Immediate publication on acceptance

- Inclusion in PubMed, CAS, Scopus and Google Scholar

- Research which is freely available for redistribution

Submit your manuscript at www.biomedcentral.com/submit
Ciomed Central 